\title{
Algunos orientalismos del Recetario para diversas enfermedades atribuido a Arnau de Vilanova
}

\author{
Mar Campos Souto \\ Universidade da Coruña
}

1. En diversas ocasiones voces más autorizadas que la nuestra han denunciado la actitud de buena parte de la Filología Hispánica, que a lo largo de su historia ha privilegiado el estudio de las obras tradicionalmente más estimadas por sus valores literarios y, en consecuencia, ha ignorado desdeñosamente la aproximación a textos no ficcionales. Este arrogante descuido es, si cabe, más inexcusable en el ámbito de la lexicografía, ya que la postergación de tratados científicos ha conducido a un desconocimiento casi absoluto en torno a considerables carnpos léxicos, c'e tal modo que nos encontramos ante una situación realmente paradójica ya que, como afirma el profesor Pérez Pascual, a partir de tales premisas "no nos puede sorprender que el conocimiento que tenemos del vocabulario feudal y su utilización en la esfera amatoria en el Medievo sea mayor que el que poseemos acerca de las denominaciones de la vestimenta, herramientas, elementos de construcción, hábitos alimenticios, o creencias de la época"1.

No obstante, en los últimos años los historiadores de la lengua y los estudiosos de la literatura han comenzado a vencer aquel curioso prejuicio, que silenciaba o prescindía de las manifestaciones escritas relativas a las funciones más inmediatas de la lengua y han descubierto el atractivo de ese español ceniciento que no sólo era digno de atención cuando salía a pasear en la quijotesca carreta de Las Cortes de la Muerte, sino que era igualmente acreedor de nuestro interés cuando, confinado en humildes aposentos y desprovisto de oropel, debía ocuparse de labores más cotidianas y domésticas. En este sentido, el proyecto de redacción del Diccionario médico medieval y la edición de diversos tratados medicinales del Medievo ha rescatado del olvido no pocos vocablos, contribuyendo así mismo a lograr un entendimiento ajustado de la intensidad del comercio de voces entre las lenguas peninsulares en este período. Aunque los hispanistas se han aplicado con mayor afán a la tarea de dar a conocer las magnas obras de los galenos medievales, no es menos cierto que el análisis detallado de textos menores se ha revelado como una fuente extremadamente fecunda desde el punto de vista lingüístico; en este sentido, el Recetario para diversas enfermedades, opúsculo que

1 Cf. José Ignacio Pérez Pascual (1993), p. 749; vid. también Ma Nieves Sánchez González de HERRERO (1992), p. 151. 
por su carácter misceláneo podría pasar fácilmente inadvertido, proporciona una valiosa información en cuanto a la introducción de préstamos del catalán, aragonés e italiano en la literatura médica castellana de la Edad Media.

2. El Recetario para diversas enfermedades ${ }^{2}$, conservado en el manuscrito b-IV-31 de la Biblioteca del Monasterio de El Escorial, se compone de cuarenta recetas en castellano, en las que se expone el modo de preparación y el correcto uso de compuestos farmacéuticos, hierbas con propiedades médicas o ungüentos para combatir diversas dolencias de hombres y bestias. Los al menos catorce amanuenses responsables de la compilación de remedios incluyen, al tiempo, fórmulas en catalán (fols. 66v y 67r), italiano (fols. 64v-66v y 68r), así como una interesante oración latina a San Sebastián. A pesar de la atribución a Arnau de Vilanova, la carencia del recurso a la auctoritas y de explicaciones minuciosas acerca de los síntomas o la evolución de los procesos morbosos sugieren que nuestro Recetario debe de ser una de tantas recopilaciones de recetas de las que se servían en el Medievo los que practicaban el arte de sanar alejados de los templos del saber científico.

3. La construcción del edificio de la medicina hispana bajomedieval habría sido imposible sin la ingente labor acometida por los intelectuales de la Corona de Aragón, cuyo peso en la literatura científica de este período se ha plasmado no sólo en el notable número de escritos redactados originalmente en catalán (o vertidos desde el latín a este idioma), sino también en la huella indeleble que los romances del Oriente peninsular dejaron en ciertas obras castellanas. En el presente trabajo nos proponemos tan sólo dar noticia de algunos de los 39 orientalismos ${ }^{3}$ que salpican este apócrifo arnaldiano y que constituyen, sin duda, una reducida muestra del alcance y trascendencia de los contactos entre castellano, catalán y aragonés en la Edad Media ${ }^{4}$.

2 Para la descripción del manuscrito, véanse nuestros El Recetario para diversas enfermedades atribuido a Arnau de Vilanova. Edición y estudio, Universidade da Coruña, 1994 (tesis de licenciatura inédita) y “Las glosas del Recetario para diversas enfermedades atribuido a Arnau de Vilanova”, en Scripta philologica in Honorem M. Taboada Cid (en prensa).

3 En 1974 José Antonio Pascual acuñó el término orientalismos para referirse a aquellos vocablos procedentes del aragonés, catalán y occitano empleados por el traductor en la versión castellana del Infierno de Dante; el insigne profesor salmantino reconocía en su excelente estudio que la imposibilidad de adscribir una voz a la norma lingüística catalana o aragonesa le había impulsado a preferir esta denominación a otras acaso más delimitadas (vid. José Antonio PASCUAL RodríGuez (1974), p. 119).

4 Indicaremos a continuación las abreviaturas más utilizadas a lo largo del presente trabajo: DCECH (Joan Corominas y José Antonio Pascual (1980-1991): Diccionario Crítico Etimológico Castellano e Hispánico, Madrid, Gredos, 6 vols.), DECLC (Joan Coromines (1980): Diccionari Etimológic i Complementari de la Llengua Catalana, Barcelona, Curial, 10 vols.), DCVB (Antoni M. Alcover y FRANCESC B. DE Moll (1993): Diccionari catalá-valenciá-balear, Palma de Mallorca, Moll), DME (Martín Alonso (1986): Diccionario medieval español, Salamanca, Universidad Pontificia), EI (Martín Alonso (1986): Enciclopedia del idioma, Madrid, Aguilar, $2^{a}$ edición), DHLE (REAl ACADEmia EsPañola (desde 1960): Diccionario histórico de la lengua española, Madrid, R.A.E), LHM (BERnARD PotTier (1980, 1981, 1982, 1983, 1984, 1987, 1992): “Lexique Médiéval Hispanique”, CHLM, 5, pp. 195-247, 6, pp. 179-217, 7, pp. 135-152, 8, pp. 197-209, 9, pp. 177-187, 12, pp. 5-26, 16, pp. 53-101), TL 


\section{ALAMBICH}

“Toma los rauanos con sus fullas e coze los vn dia antes e despues pica los bien e mete los en vn alambich" (7r).

Esta voz (procedente del árabe anbiq) y sus variantes alanbich o alambichs se registran, amén de en otros textos médicos ${ }^{5}$, en inventarios aragoneses de fines del siglo $\mathrm{XV}^{6}$, en los Libros de Collidas ${ }^{7}$ y en ciertas obras catalanas ${ }^{8}$. Si bien J. A. Sesma aseguran que alanbich es forma aragonesa (y alambich catalana o valenciana) ${ }^{9}$, la documentación existente no parece autorizar por completo esta aseveración, ya que M. Serrano y Sanz recoge alambich en inventarios aragoneses de 1380 y $1487^{10}$.

\section{$\operatorname{CREMA}(L A)$}

"\& rae [...] la dicha vexjga e crema la" (16r).

El verbo cremar (del latín CRĔMĀRE), común a todos los dialectos catalanes y general a todos los tiempos ${ }^{11}$ es, en cambio, voz rara en las restantes lenguas románicas

(Samuel Gili Gaya (1957): Tesoro lexicográfico, Madrid, C.S.I.C.). Los textos médicos empleados son: CAU (Guido de Caulicaco: Tratado de cirurgia, ms. I-196 de la Biblioteca Nacional de Madrid -citamos según la transcripción de Juan GutiérRez Cuadrado-), CHI (Alfonso Chirino (1987): The Texts and Concordances of Escorial Manuscript b.IV.34. Menor daño de medicina by Alfonso Chirino, ed. de E. J. Ardemagni et al., Madison, Hispanic Seminary of Medieval Studies), CHS (JohANNES DE KETHAM (1987): Compendio de la humana salud, I-51, Biblioteca Nacional, Madrid, Madison, Hispanic Seminary of Medieval Studies), FLO (Fernando DE CóRdoBA (1987): The Text and Concordance of the Suma de la Flor de Cirugía, Biblioteca Nacional, Madrid, 3383, edición de $\mathrm{M}^{a}$ Carmen Villar, Madison, Hispanic Seminary of Medieval Studies), FOR (LiCEnCIADo Forés (1987): The Text and Concordance of Biblioteca Nacional MS. I.51, Tratado util, ed. de M. P. Zabía, Madison, Hispanic Seminary of Medieval Studies), MAC (Text and Concordance of Seville Colombina Manuscript 7-6-27 Macer Herbolario, ed. de P. Conerly y otros, Madison, Hispanic Seminary of Medieval Studies, 1986), RES (Recetas. Biblioteca Universitaria, Salamanca, 2262, ed. Ma C. de la Cal, Madison, Hispanic Seminary of Medieval Studies, 1987), SEV (J. DE AvIÑón (1987): Sevillana medicina, Burgos, 1545, ed. de E. W. Naylor, Madison, Hispanic Seminary of Medieval Studies), SUM (F. LóPEZ DE VILLALobos (1987): Sumario de la medicina, 1-1169, Biblioteca Nacional, Madrid, Madison, Hispanic Seminary of Medieval Studies) y TRM (Tratado de patología general. Biblioteca Nacional, Madrid, 10.051, ed. de $\mathrm{M}^{\mathrm{a}} \mathrm{T}$. Herrera, Madison, Hispanic Seminary of Medieval Studies, 1987).

5 Alanbich en CAU 64v45.

6 Vid. Manuel Serrano y Sanz (1915 y 1916), pp. 89 y 359 y Bernard Pottier (1948-1949), p. 94.

7 Alanbich en Daroca y Barracas (Vid. J. Á. Sesma y Á. Líbano (1982), p. 71).

8 Alambichs en Lo Somni de Bernat Metge y alambich en un documento fechado hacia 1523. (Vid. DCVB, s. v. alambí o alambic).

9 Vid. J. A. Sesma y Á. Líbano (1982), p. 71.

10 Vid. M. Serrano y Sanz (1915 y 1916), pp. 89 y 359.

11 Diferentes variantes de cremar figuran en los autores paradigmáticos de la literatura medieval catalana: Jaume I, Ramón Llull (crema-cremar en el Felix de les Meravelles del Mon), Ramón Muntaner (cremaren en la Crónica); como sinónimo de cauterizar lo emplean F. Eiximenis, Mossén Manuel Dieç y Joan Martina (Vid. DCVB y DECLC, s. v. cremar, J. Perarnau I Espelt (1992), pp. 304, 308, 309. etc. y L. Faraudo DE SaINT GeRmain (1955-1956), p. 17, etc.). 
(excepto en occitano, lengua en la que la emplean ya los primeros trovadores). No obstante, Joan Corominas y Martín Alonso atestiguan también su presencia en obras medievales castellanas ${ }^{12}$; es, además, vocablo familiar al aragonés antiguo ${ }^{13}$ y hoy pervive en las hablas del Alto Aragón ${ }^{14}$.

\section{ESCORÇA-ESCORCHA}

"\& toma el sabuco \& raelo sotil \& aneta la escorça de fuera" (6r).

"tomaras la toronja qua $<\mathrm{n}>$ to mas fresca la podras hauer, e tajada la escorcha" (8v).

La riqueza léxica de nuestro texto se manifiesta con especial intensidad en la concurrencia de cuatro de las voces que se conocen en el ámbito románico para designar la corteza: además de las ya mencionadas escorça y escorcha, los copistas del Recetario para diversas enfermedades usan cortezas ${ }^{15}$ y scorza ${ }^{16}$. El catalanismo escorça ${ }^{17}$ (común al occitano escorsa, al francés écorce, al rumano scoarta y al italiano scorza) se documenta en este idioma desde mediados del siglo XIII, al igual que su variante escorxa, extendida hoy a los dominios del catalán occidental, insular y meridional ${ }^{18}$. Aun-

12 Joan Corominas y Martín Alonso aseguran que este verbo no es totalmente ajeno al castellano medieval, puesto que se registra en el Fuero Juzgo y en el Fuero de Avilés (en el que acaso sea un occitanismo). (Vid. El, s. v. cremar; DCECH, s. v. quemar y V. FeRnÁNDEZ LleRA (1929), p. 146.

13 En un inventario aragonés de 1379 y en un documento de 1502 conservado en el Archivo Municipal de Jaca; vid. DCECH, s. v. quemar y M. AlVAR (1960), p. 130.

14 Rafael Andolz asegura que cremá se usa en aragonés como transitivo con el significado de "arder, quemar" en Estadilla, Puebla de Roda y Ribagorza, en tanto que cremar es sinónimo de "quemar, socarrar" en Biescas y Echo. Del mismo modo, A. Badía Margarit registra los verbos Kremar ("quemar" en Bielsa) y kremá ("quemar, incendiar", en Benasque y únicamente "quemar" en Peralta), al tiempo que advierte un uso ocasional con el sentido de "chamuscarse la comida"; el mismo autor afirma que en el Valle de Bielsa cremar conoce las acepciones "quemar, encender, abrasar". En Benasque, según A. Ballarín, kremá se emplea como transitivo o reflexivo con el significado de "encender", mientras que como intransitivo equivale a "estar encendido, consumirse con el fuego". A su vez, A. Viudas Camarasa documenta cremáda en la zona de la Litera. (Vid. R. Andolz (1977), s. v. cremá y cremar; A. Ballarín Cornel (1978), s. v. kremá; A. Badía (1950), s. v. cremá; A. Badía, (1948), p. 66; V. FERRAZ y CAstán (1934), p. 43 y A. Viudas Camarasa (1983), p. 70).

15 "Toma de las cortezas de las granadas" $(2 r)$.

16 “Toma la scorza del salz e talla la bien men<u>da" (8r).

17 Escorza se registra en ciertos textos castellanos de influencia dialectal, como en CHS 34r 15. Al tiempo, escorchar figura en los Ordenamientos y posturas y otros capítulos generales otorgados a

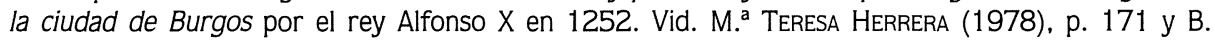
POTTIER, (1983), p. 208.

18 Escorça en los Proverbis de Guillem de Cervera, en el Maçer de fines del XIV, en el Spill de Jacme Roig y en el Breviari d'Amor; scorces en el Inventari o collectori en la part cirugical de medicina de G. de Chauliac; scorça en Lo Somni de Bernat Metge y escorxa en los Proverbis de Guillem de Cervera, en el Breviari d'Amor y en R. Llull; vid. DECLC, s. v. escorça; DCVB, s. v. escorça y LI. FARAUDo DE SAINT Germain (1955-1956), pp. 15 y 22). En el catalán actual, según A. Griera, escorça posee tres acepciones: "la pela més exterior dels arbres" (en Cataluña, Baleares y Valencia), "la segona pela del suro" (en Sant Feliu de Guíxols) y "la pela corretjosa dels melons i síndries" (En Banyols), (A. GrierA (1966-1970), s. v.). 
que este vocablo tuvo una cierta difusión en el aragonés antiguo, escorcha es la forma genuina y dominante en este domino lingüístico ${ }^{19}$.

\section{GINEBRO}

"olio de ginebro media <libra>" (fol. 7v).

El aragonesismo ginebro ${ }^{20}$ (del latín vulgar JINIPERUS) figura ya en los Libros de Collidas de las localidades de Alcañiz, Híjar y Monroyo ${ }^{21}$; en la actualidad pervive en territorio aragonés en Caspe, Puebla de Híjar y se extiende al dominio navarro ${ }^{22}$.

\section{MALALTIA}

\section{"Otro Remedio a la dicha malaltia" (3v).}

19 Joan Corominas recuerda el uso que el aragonés morisco del siglo XVI hizo del término eskuercha, mientras que J. A. Sesma y A. Líbano recogen las variantes escorza, escorça y ascorça en los Libros de Collidas de Zaragoza y Barracas. A su vez, Javier Terrado Pablo reconoce las variantes escorchar (documentada, del mismo modo, en unas ordenanzas aragonesas de 1488) y scorchar en un documento turolense de 1470, adviertiendo que su documentación se remonta en Aragón al principio del XIV. A principios de siglo eran de uso común, según Jerónimo Borao, escorcha ("túrdiga”) y escorchar ("levantar la corteza o piel a alguna cosa, desollar"). Manuel Alvar, a su vez, registra escorchar con el valor de "despellejar" en la Revista de Aragón de 1904, así como el verbo escorcotar (propio del Campo de Jaca). Martín Alonso introduce en su Enciclopedia del idioma los aragonesismos escorcar ("quitar la cáscara a las almendras o nueces"), escorcha ("escoriación, escorchadura"), escorchado, -da ("Se dice del lobo de color de gules que aparece desollado", uso reconocido también por E. de Terreros y Pando), escorchadura y escorchar ("levantar la corteza o piel a alguna cosa”). Rafael Andolz recoge los vocablos escorcha (usado en Benasque y Xistau con los significados "trozo de cuero verde necesario para hacer un par de abarcas" y en Peralta de la Sal como sinónimo de "escoriación”), escorchá ("despellejar, desollar”, verbo propio de Benasque y Peralta de la Sal), escorchadura y escorchar (con las acepciones "desollar, levantar la corteza o la piel de alguna cosa”, "dormir plácidamente la borrachera" -en Ayerbe, Bolea y Loarre- y "descorchar, abrir una botella" -en Huesca-). A. Ballarín reconoce el uso de eskórcha en el benasqués (como sinónimo de túrdiga, aclarando que "no significa trozo de corteza de árbol") y escorchá (verbo intransitivo con el valor "quitar el cuero, la piel a una res de ganado mayor, despellejarla. Dividir un cuero en anchas tiras. En sentido figurado, despellejar a una persona, murmurar de ella"). Vid. J. TerRado Pablo (1991), pp. 534 y 260; J. BoraO (1908), s. v.; M. Alvar (1948), p. 203; El, s. v.; E. DE Terreros y Pando (1987), s. v.; R. Andolz (1977), s. v.; A. Ballarín (1978), s. v..

20 Documentado así mismo en CHS 17r4, en el Libro de Marco Polo (fol. 29v) y en el Vocabulario eclesiástico de Rodrigo Fernández de Santaella de 1499 (fol. 100v).

21 Vid. José A. Sesma y A. Líbano (1982), p. 224.

22 En su Colección de voces usadas en la literatura de 1908, Benito Coll y Altabás caracteriza esta voz como aragonesismo, al tiempo que Jerónimo Borao recuerda que Francisco Loscos creía este término una de las denominaciones vulgares del enebro (vid. Jerónimo Borao (1908), s. v.). Para el uso y extensión actual de la voz, vid. M. Alvar (1953), p. 199. J. M. IriBARREN (1984), s. v., El, s. v., A. ZAMORA VICENTE (1989), p. 223-114, DCECH, s. v. enebro y DECLC, s. v. ginebre. En Aragón se emplean otras variantes como _inebro (Jaca), chinebro (Ansó, Echo, la Litera, Aragüés, Embún, Fiscal, Plan, Torla e Ispiés), chinepro (Sallent, bolea, Loarre, Bielsa, Biescas), chinebre (Xistau, Ribagorza), inebro (Alcalá de la Selva), chinebrero (Alquézar) y chenebro (Buerba). 
Estamos ante un catalanismo (derivado del adjetivo malalt ${ }^{23}$ ) que apenas conoce uso entre los escritores de lengua castellana ${ }^{24}$, quienes optan usualmente por préstamos del italiano (malatia o maletia) o del occitano (malautía) ${ }^{25}$. En catalán es, sin embargo, voz arraigada y viva en todo su dominio lingüístico ${ }^{26}$, así como en dialectos vecinos ${ }^{27}$.

\section{MALVASÍA} $(4 r)$.

"toma vna taça del mejor vino q<ue> trouaras et si ser puede greco, o, maluasia"

La denominación de este vino, considerado un caldo de extraordinaria calidad en la Edad Media, es con toda probabilidad un catalanismo en castellano ${ }^{28}$. El aragonés

23 Del latín vulgar MALE HABITUS (participio de MALE SE HABERE). En principio evolucionó a malaute y después a malalte; la ulterior reducción a malalt es de naturaleza analógica. (Vid. DECLC, s. v. malalt).

24 No obstante, se registra en el Tratado de Cetrería de Ayala y en obras de marcado cariz oriental, como la Triste deleytaçión, el Llibre del arte de menescalia (fols. 3v, 4r, etc.), el Libro de las maravillas del mundo y el Compendio de la humana salud (en el incunable zaragozano de 1494). Vid. R. Ma Gómez Fargas (1989), pp. 54-55, M. ${ }^{a}$ T. HerRera (1978), p. 172 y J. Terlingen (1943), p. 356.

${ }^{25}$ Malatía, voz usada en el castellano medieval con las acepciones de 'lepra, gafedad' o 'enfermedad' y documentada en el Libro del caballero Zifar, Calila e Dimna, Libro de Apolonio, Vida de Santo Domingo de Silos, las Siete Partidas y el Fuero de Cuenca (1284-95), es, a juicio de CorominasPascual, un italianismo temprano que se justifica por la abundancia de leprosos en el Levante, en tanto que malautía (Libro de Alexandre, TRM 30r38) ha de ser occitanismo. En español se conocieron también las variantes maletía (en Berceo, FLO 180v46 y MAC 19v18) y malaudía (Fuero de Navarra), amén de otras voces emparentadas (malatería -usada entre los siglos XV al XX-, malato -en el Recetario para diversas enfermedades-, etc.).

26 Malaltia en el Somni de Bernat Metge, en un documento de 1299, en el Regiment de presevació; maletia en R. Llull y malautia en un texto del XIV, en Jaume I, etc. (Vid. DECLC y DCVB, s. v. y J. Perarnau i Espelt (1992), p. 312)

27 En benasqués se conocen las voces malutiá (verbo intransitivo, con el significado 'sentir un poco quebrantada la salud') y malutía (sustantivo, sinónimo de 'enfermedad'). Justo García Soriano afirma, a su vez, que maletía se usaba a principios de siglo en la zona de Lorca para designar los procesos morbosos.; vid. A. Ballarín (1978), s.v. malutía y malutiá y J. GarCía Soriano (1932), s. v. maletía.

28 En catalán malvesia se documenta desde 1403, en el Tirant de Joanot Martorell, en el Spill de J. Roig y en el Regiment preservartiu e curatiu de la pestilencia de L. Alcanyís (1940); vid. DECLC y $D C V B$, s. v. malvasia.

Aunque Martín Alonso asegura que esta voz se usó en castellano entre los siglos XVI y XX para nombrar esta clase de uva (por Gabriel Alonso de Herrera) -y que sólo a partir del siglo XVI se conoce para referirse al vino elaborado con esta uva (así lo hace Leandro Fernández de Moratín)- y Corominas-Pascual cifran la primera documentación de esta voz en el siglo XVI, los textos médicos nos deparan testimonios más antiguos (maluasias en SUM, maluasia en la Cura de la piedra y dolor de la ijada y cólica renal -fol. 28v-o incluso en escritos de viajes como la Peregrinatio in Terram Sanctam. Viaje siquier peregrinación de la tierra sancta-fol. 178r-). Como afirman los ilustres lexicógrafos, en castellano debió de tomarse del catalán, dado que los catalanes trajeron esta cepa de Quío durante su dominación en Grecia en el siglo XIV. Terreros dice que también se le daba este nombre, además de 
medieval no ignoraba en absoluto este vocablo, como lo atestigua la presencia de malvasía en los Libros de Collidas de Zaragoza ${ }^{29}$.

\section{METRIDAT} (1r).

"e tome de la triaca en quantidat de vna cucharada e otra cucharada de metridat"

"darle çinco cucharadas de cada agua e son todas q<ui>nze e dos cucharadas de metridat/ e si fuere de quinze, tres de cada agua e media cucharada de triaca e media de metridat" (1v).

La proverbial afición al estudio de los venenos de Mitrídates VI Eupátor, rey del Ponto, muerto en el año 65 a. C., dio nombre a este electuario. Las formas catalanas metridat ${ }^{30}$ y mitridat alternan en las obras catalanas y aragonesas con materdat ${ }^{31}$ y matridat ${ }^{32}$.

\section{POLVORA}

"e, en la maniana dale de vna gallina asada e, su vino e, en la noche la suso dicha cena e a la media noche la otra meitat de la poluora segunt ya es scripto" (5r).

"\& en aquella poluora baña el dedo" (9r).

Si bien en principio Corominas-Pascual no rechazan radicalmente la hipótesis de la posible procedencia occitana de esta voz (del latín *PǓLVĚRA), conceden mayor autoridad al origen catalán ${ }^{33}$ por motivos de índole histórico y lingüístico ${ }^{34}$. Germán

al vino griego o de Candía, a un "vino estimable que viene de Provenza”. (Vid. DCECH, s. v. malvasía; El, s. v. malvasía y E. DE TerReros y Pando (1987), s. v.).

29 Vid. J. A. Sesma y A. Líbano (1982), p. 248 y M. Gual Camarena (1976), p. 355). Según J. Terrado Pablo, el hecho de que los catalanes trajeran la cepa explica la introducción del término en Aragón, registrado en diversos documentos emitidos por la cancillería de la Corona. Por su parte, Jerónimo Borao discrepaba de la doctrina emanada de la Real Academia Española (que consideraba esta voz propia del español, dado que había sido usada por autores castellanos, como Castillejo) y juzgaba malvesía como un aragonesismo, basándose en un curioso testimonio de Avellaneda ("Tengo en el cuerpo 3 de MALVASÍA que llaman en esta tierra -en Zaragoza- y á fé con razón, porque está MALA la taza, cuando está VACíA"). Vid. J. TerRado Pablo (1991), p. 317 y J. Borao (1908), s. v.

30 En L. Alcanyís, Regiment preseruatiu e curatiu de la pestilencia.

31 En el inventario de una botica de Zaragoza del 3 de diciembre de 1488; vid. M. SERRAno y SAnZ, (1922), p. 128.

32 En los Libros de collidas de Zaragoza.; vid. J. A. Sesma y A. LíBano (1982), p. 254.

33 Polvera figura en las Vides de sants roselloneses, en las Ordinacions del Vall d'Aneu de 1424 y en diversos documentos del siglo XIV; polvora se registra en este idioma desde fines del siglo XIII, en Ramón Llull, el Tirant de Joanot Martorell, el Receptari de Joan Martina y el Macer de fines del XIV. Vid. DECLC, s. v. pols; DCVB, s. v. polvora; J. Perarnau I Espelt (1992), pp. 301, 304 y 309 y Ll. Faraudo de Saint-Germain (1955-1956), pp. 11, 16, 19, etc.

34 De hecho, aunque polvera existía en occitano antiguo desde principios del siglo XIII, pronto fue sustituida por variantes como poldra, pobra y porba, explicables por la resistencia de este idioma a los proparoxítonos. ( $D C E C H$, s. v. polvo). El cambio de polvera a polvora se justifica, según Joan Coromines, debido a la asimilación de e a la o tónica, fenómeno propio del catalán; vid. DECLC, s. v. pols y JOAN COROMINES (1993), pp. 199 y 296. 
Colón ${ }^{35}$ insiste la tesis catalana (única explicación factible a su juicio desde el punto de vista fonético) y desecha por completo la hipótesis occitana; para este investigador la utilización del término en castellano con la acepción secundaria de 'mezcla explosiva de salitre, azufre y carbón', frente al valor general del catalán, confirma su carácter adventicio. No obstante, Corominas-Pascual recogen la afirmación de la Academia, que asegura que pólvora tuvo en castellano el sentido general de "polvos", aunque los autores del $D C E C H$ consideran que debió de ser bastante raro y admiten que sólo en el idioma del Oriente peninsular fue general este uso. En realidad, la acepción 'polvos usados con finalidad medicinal' es ciertamente frecuente en los textos médicos medievales castellanos, como lo demuestra el propio Recetario para diversas enfermedades $^{36}$; con este valor lo registran también J. A. Sesma y A. Líbano en los Libros de Collidas de Zaragoza, aunque en los textos aragoneses figura generalmente con la acepción bélica.

\section{POLVORIZAR}

"e despues toma gengiure polvoriçado" (4v).

"e fazer lo as bien polvorizar todo" (5r).

El catalán polvoritzar ${ }^{37}$ se halla en el origen del castellano polvorizar ${ }^{38}$, que posteriormente sufrió una refacción culta en pulverizar.

\section{RAJOLA}

“olio de rajola media <libra>" (7v).

M. Aguiló, al documentar la expresión oli de rajolas en un escrito farmacéutico de 1736, proponía con ciertas reservas el significado "una planta" para este catalanismo de raíz árabe. Acaso influido por esta opinión, A. Griera incluye rajola en su Tresor de la llengua con la acepción "nom de planta". No obstante, A. Alcover y F. de Moll desvelan que nos hallamos ante un fantasma lexicográfico, al retomar un fragmento del

35 Vid. G. Colón (1967), p. 225. Ralph Penny coincide con Corominas-Pascual y G. Colón en defender el origen catalán del término (Vid. R. PENNy (1993), p. 252).

36 Así en CHI 41v8, FLO 167v31, MAC 11v36, CAU 31v128, RES 27r4, FOR 4v5, CHS 34r75 y Llibre del arte de menescalia (fol. 17v), Cura de la piedra y dolor de la ijada y cólica renal (fols. 36r, $44 r . .$.$) , etc. Corominas-Pascual creen que el sentido general debe de ser bastante raro en castellano,$ puesto que falta totalmente en el Diccionario de Autoridades, en Covarrubias, Oudin o Nebrija y tan sólo lo recoge Alonso de Palencia. Martín Alonso documenta el valor 'polvos con fines medicinales' en el Pedacio Dioscórides de Laguna, en tanto que Bernard Pottier y Manuel Serrano y Sanz reconocen este uso en ciertos inventarios aragoneses. (Vid. El, s. v., M. SERRAno y SANZ (1922), pp. 126 y 131 y B. PotTier (1948-1949), p. 193).

37 Diferentes variantes de este verbo figuran en un documento rosellonés de 1398, en el Spill de Jacme Roig, en el Somni de Bernat Metge y en el Receptari de Joan Martina. (Vid. DECLC, s. v. pols, DCVB, s. v. polvoritzar (polvorisar) o pulverizar y J. PeraRnau I ESPELT (1992), p. 320).

38 Documentado en Alonso de Palencia, Robert de Nola, Libro de albeyteria -o Llibre del arte de menescalia, fol. $24 r-$ etc. Martín Alonso asegura que se usa desde el siglo XVI con el sentido de 'polvorear'. (Vid. $E I$, s. v.). 
Libre de Menescalia de Mosén Manuel Dieç (1523), en el que se describe el proceso de elaboración de este compuesto, hecho a base de aceite de oliva y polvo de ladrillos ${ }^{39}$. Este arabismo, propio del catalán ${ }^{40}$, penetró en fecha temprana en aragonés, idioma en el que se documenta desde $1350^{41}$.

\section{SINSE}

"beuer cada semana el brodo de los faysoles sinse sal \& sin olio" ( $3 \mathrm{v})$

La preposición aragonesa sinse ${ }^{42}$ (del latín SINE más -s adverbial), figura en considerables documentos medievales ${ }^{43}$; si bien es voz extraña al diasistema catalán, está emparentada con el catalán sense y ciertas voces de otros romances (como el occitano antiguo sense y el italiano, de origen incierto, senza $a^{44}$ ).

39 "En quina manera se deu fer lo oli de les rajoles... Preneu rajoles fresques cuytes vermelles quant hixien del forn, que aygua no les haja tocades, e picau-les en feu-ne pólvora... Meteu-las en un llibrell net e pastau-la ab oli de olives... Preneu un fornet de terra e meteu dintre la dita pasta fresca... e aprés donau foch de carbó al dit fornet". (Cf. $D C V B$, s. v. rajola).

40 Del hispanoárabe lagura (y éste del árabe clásico al-'agú $(r) r$ ), rajola figura en diversos documentos de los siglos XIII, XIV y XV, en diversas obras de F. Eiximenis y F. Roig. (DECLC y DCVB, s. v. rajola).

41 J. A. Sesma y A. Líbano recogen las variantes rejolleta y ragolas en los Libros de Collidas de Tarazona, Zaragoza y Barlena, en tanto que G. Tilander registra la forma regola en los fueros aragoneses posteriores a 1348. Rajola figura además en el documento Nuevas instrucciones de los regidores al maestro de construcción del Puente del Vado de 1468, en la que, según Javier Terrado Pablo, debe ser un catalanismo difundido por el vocabulario propio de la construcción. (Vid. J. A. Sesma y A. Líbano (1982), p. 308, G. Tilander (1935), p. 25 y J. Terrado Pablo (1991), pp. 297 y 539). Aunque Rafael Andolz da regola como anticuada en su Diccionario aragonés, en la actualidad este vocablo pervive en parte del territorio aragonés para designar un tipo de material usado para el pavimento (rajola en Valderrobres y reijola en Peñarroya de Tastavíns) y como material de construcción (rajola en Valderrobres); vid. R. Andolz (1977), s. v. regola y R. M. ${ }^{a}$ CASTAÑER MaRTín (1990), pp. 27-28.

$42 \mathrm{Si}$ bien las formas con $i$ no están suficientemente explicadas (acaso se deban a un cruce con sino o nin), la $s$ adverbial se debió adherir a la preposición en el latín tardío, a causa de la frecuencia de combinaciones del tipo sine-s-terra. ( Vid. DECLC, s. v. sense y M. Alvar y B. PotTIER (1987), p. 294).

43 Sinse figura en inventarios de 1487 y 1497, en un documento de 1471, así como en algunos textos castellanos de notable influencia aragonesa, como la Triste deleytaçión. ( $D C E C H$, s. v. sin y R. M. ${ }^{a}$ Gómez-FarGas (1989), p. 43). R. Andolz la considera un arcaísmo en el aragonés moderno (Vid. R. Andolz (1977), s. v.).

44 Joan Coromines asegura que aunque esta variante haya sido caracterizada ocasionalmente como propia del valenciano, su uso por autores de lengua catalana debe ser interpretado como barbarismo. Para las explicaciones acerca de esta voz, vid. $D C E C H$, s. v. $\sin$; $D E C L C$, s. v. sense y J. Corominas (1972), p. 92. 
BiBLIOGRAFÍA

Alcover, Antoni y Francesc B. De Moll (1993): Diccionari català-valencià-balear, Palma de Mallorca, Moll, 10 vols.

Alfonso ChiRINo (1984): The Texts and Concordance of Escorial Manuscript b. VI.34. Menor daño de medicina by Alfonso Chrino, ed. de E. J. Ardemagni et al., Madison, Hispanic Seminary of Medieval Studies.

Alfonso de Palencia (1992): Universal vocabulario en latín y romance, en ADMYTE. Archivo digital de manuscritos y textos españoles, transcripción de Gracia Lozano, $\mathrm{M}^{\mathrm{a}}$ Dolores Fuentes Portillo, $\mathrm{M}^{\mathrm{a}}$ Ángeles Lamolda González, Cristina Ceballos Rodríguez, Carmen Ortega Alcaraz y Ángel Romero Díaz, Madrid, Micronet-Quinto Centenario- Ministerio de Cultura. Biblioteca Nacional.

Alonso, MarTín (1986): Diccionario medieval español, Salamanca, Universidad Pontificia de Salamanca, 2 vols.

Alonso, MarTín (1982): Enciclopedia del idioma, Madrid, Aguilar.

Alvar, Manuel (1960): Documentos de Jaca (1361-1500), Zaragoza, Instituto Fernando el Católico.

Alvar, Manuel (1953): El dialecto aragonés, Madrid, Gredos.

Alvar, Manuel (1948): El habla del Campo de Jaca, Salamanca, C.S.I.C.

Alvar, Manuel y Bernard PotTier (1987): Morfología histórica del español, Madrid, Gredos.

Álvarez, FeRnando (1992): Regimiento contra la peste, en ADMYTE. Archivo digital de manuscritos y textos españoles, I, transcripción de Guadalupe Rodríguez López-Lago, Madrid, Micronet-Quinto Centenario-Ministerio de Cultura. Biblioteca Nacional.

Andolz, Rafael (1977): Diccionario aragonés, Zaragoza, Librería General.

Badía MaRGaRIT, Antonı (1948): Contribución al vocabulario aragonés moderno, Zaragoza, Monografías de la Estación de Estudios Pirenaicos.

Badía Margarit, Antonı (1950): El habla del valle de Bielsa (Pirineo aragonés), Barcelona, Instituto de Estudios Pirenaicos.

Ballarín CoRnel, Ángel (1978): Diccionario del benasqués, Zaragoza, Talleres Gráficos la Editorial, $2^{\mathrm{a}}$ ed.

Ballarín CoRnel, Ángel (1974): “El habla de Benasque”, Revista de dialectología y tradiciones populares, 30, pp. 99-215.

Ballarín CoRnel, Ángel (1979): Vocabulario de Benasque, Zaragoza, Institución Fernando el Católico.

BREYDENBACH, BeRnhaRd von (1992): Peregrinatio in Terram Sanctam. Viaje siquier peregrinación de la tierra santa, en ADMYTE. Archivo digital de manuscritos y textos españoles, I, transcripción de Pedro Tena Tena, Madrid, Micronet-Quinto Centenario-Ministerio de Cultura. Biblioteca Nacional.

Cal, Ma del Carmen de la, ed., (1987): Recetas. Biblioteca Universitaria, Salamanca, 2262, Madison, Hispanic Seminary of Medieval Studies.

CAmpos Souto, MaR: "Las glosas del Recetario para diversas enfermedades atribuido a Arnau de Vilanova", Scripta philologica in honorem M. Taboada Cid (en prensa). 
Algunos orientalismos del Recetario para diversas enfermedades...

Castañer Martín, Rosa María (1990): Estudio del léxico de la casa en Aragón, Navarra y Rioja, Zaragoza, Diputación General de Aragón.

CASTRo, Américo (1921, 1922, 1923): “Unos aranceles de aduanas del siglo XIII”, RFE, 8, pp. 129 у 325-356; 9, pp. 266-276 у 10, pp. 133-136.

ConerLy, P. et al., eds., (1987): Text and Concordances of Seville Colombina Manuscript I-6-27 Macer Herbolaria, Madison, Hispanic Seminary of Medieval Studies.

Colón DOMÉNECH, GERMÁn (1967): “Elementos constitutivos: catalanismos", Enciclopedia lingüística hispánica, II, Madrid, C.S.I.C., pp. 193-238.

Colón Doménech, Germán (1967): "Elementos constitutivos: occitanismos", Enciclopedia lingüística hispánica, II, Madrid, C.S.I.C., pp. 153-192.

Corominas, Joan y José Antonio Pascual (1980-1991): Diccionario crítico etimológico castellano e hispánico, Madrid, Gredos.

CoRominas, Joan (1972): Tópica Hespérica. Estudios sobre los antiguos dialectos, el sustrato y la toponimia romances, Madrid, Gredos, 2 vols.

COROMINAS, JoAn (1980): Diccionari etimológic i complementari de la llengua catalana, Barcelona, Curial.

Corominas, Joan (1993): Lleures i converses d'un filóleg, Barcelona, Club Editor, $3^{\mathrm{a}}$ ed.

Díaz, Mossén Manuel (1530): Libro de albeytería, Burgos, Juan de Junta.

Díez de Calatayud, Manuel (1992): Llibre del arte de menescalia. Libro de albeitería, transcripción de Antonio Cortijo, en ADMYTE. Archivo digital de manuscritos y textos españoles, Madrid, Micronet-Quinto Centenario-Ministerio de Cultura. Biblioteca Nacional.

Faraudo de SaINT-Germain, Lluís (1955-1956): “Una versió catalana del Libre de les herbes de Macer", Estudis Románics, 4, pp. 1-54.

FernándeZ de LleRA, Victor (1929): Gramática y vocabulario del Fuero Juzgo, Madrid, R.A.E.

FernándeZ de SAnTAella, Rodrigo (1992): Vocabulario eclesiástico, en ADMYTE. Archivo digital de manuscritos y textos españoles, I, transcripción de Gracia Lozano, María Dolores Fuentes Portillo y $\mathrm{M}^{\mathrm{a}}$ Ángeles Lamolda Gonz

Fernando de CóRdoba (1987): The Text and Concordance of the Suma de la Flor de Cirugía, Biblioteca Nacional, Madrid, 3383, ed. de Carmen Villar, Madison, Hispanic Seminary of Medieval Studies.

FerRAZ y CASTÁn, VicenTe (1934): Vocabulario del dialecto que se habla en la Alta Ribagorza, Madrid.

García Soriano, Justo (1932): Vocabulario del dialecto murciano, Madrid.

Gómez FarGas, Rosa María (1989): "Peculiaridades lingüísticas aragonesas en Triste deleytaçion", Archivo de Filología Aragonesa, 42-43, pp. 21-64.

Gili Gaya, Samuel (1957): Tesoro lexicográfico, Madrid, C.S.I.C.

GrieRA, A. (1966-1970): Tresor de la llengua, de les tradiciones $i$ de la cultura popular de Catalunya, Barcelona, Polígrafa, $2^{\mathrm{a}}$ ed., 14 vols.

Gual Camarena, Miguel (1976): Vocabulario del comercio medieval, Barcelona, El Albir. 
GutiéRREZ dE Toledo, JuLIÁn (1992): Cura de la piedra y dolor de la ijada y cólica renal, en ADMYTE. Archivo digital de manuscritos y textos españoles, I, transcripción de Linda S. Lefkowitz, Madrid, Micronet-Quinto Centenario-Ministerio de Cultura. Biblioteca Nacional.

Herrera, María Teresa (1978): “El Compendio de la salud humana. Estudio dialectal", Studia Philologica Salmanticensia, 2, pp. 159-176.

HerRera, María Teresa (1987): Tratado de patología general. Biblioteca Nacional, Madrid, 10.051, Madison, Hispanic Seminary of Medieval Studies.

IribarRen, José María (1984): Vocabulario navarro, Pamplona, Comunidad Foral de Navarra. Institución Príncipe de Viana.

Johannes de KeTHAm (1987): Compendio dela humana salud, I-51, Biblioteca Nacional, Madrid, ed. de María Teresa Herrera, Madison, Hispanic Seminary of Medieval Studies.

JohanneS De Ketham (1992): Fasciculus medicinae, Compendio de la salud humana, en ADMYTE. Archivo digital de manuscritos y textos españoles, transcripción de Michael T. Ward y María Teresa Pajares Giménez, Madrid, Micronet-Quinto Centenario-Ministerio de Cultura. Biblioteca Nacional.

JuAn DE Aviñón (1987): Sevillana medicina, Burgos, 1545, ed. de E. W. Naylor, Madison, Hispanic Seminary of Medieval Studies.

LICENCIADO Forés (1987): The Text and Concordance of Biblioteca Nacional MS. I. 51, Tratado util, ed. de M. P. Zabía, Madison, Hispanic Seminary of Medieval Studies.

López de VILlalobos, F. (1987): Sumario de la medicina, l-1169, Biblioteca Nacional, Madrid, ed. de $M^{a}$ Nieves Sánchez, Madison, Hispanic Seminary of Medieval Studies.

Marco Polo (1992): Libro de Marco Polo, en ADMYTE. Archivo digital de manuscritos y textos españoles, I, transcripción de José Carlos Pino Jiménez, Madrid, Micronet-Quinto Centenario-Ministerio de Cultura. Biblioteca Nacional.

Pascual Rodríguez, José Antonio (1974): La traducción de la Divina Commedia atribuida a $D$. Enrique de Aragón. Estudio y edición del infierno, Salamanca, Universidad de Salamanca.

Penny, Ralph (1993): Gramática histórica del español, Barcelona, Ariel.

Perarnau I Espelt, J. (1992): “El Receptari del sabadellenc Joan Martina (1439). (Capentràs, Biblioteca Inguimbertine, ms. 126, ff. 86-90, etc.", Arxiu de Textes Catalans Antics, 11, pp. 289-328.

Pérez Pascual, José Ignacio (1993): “Algunas aportaciones de la Suma de la Flor de Cirugía al conocimiento del léxico medieval castellano", en José Antonio Bartol Hernández et al., eds., Estudios filológicos en homenaje a Eugenio de Bustos Tovar, Salamanca, Universidad de Salamanca, pp. 749-760.

PotTIer, BernaRd (1948-1949): "Etude lexicologique sur les Inventaires aragonais", Vox Romanica, 10, pp. 87-219.

Pottier, Bernard (1980, 1981, 1982, 1983, 1984, 1987, 1992), “Lexique Médiéval Hispanique”, CHLM, 5, pp. 195-247, 6, pp. 179-217, 7, pp. 135-152, 8, pp. 197-209, 9, pp. 177187, 12, pp. 5-26, 16, pp. 53-101.

Real Academia Española (desde 1960): Diccionario histórico de la lengua española, Madrid, R.A.E. 
SÁnchez González de HerRero, Ma Nieves (1990): “Nombres de composiciones farmacológicas formados con la partícula griega DIA contenidos en obras médicas medievales", RFR, 7, pp. $151-173$

Serrano y Sanz, Manuel (1915, 1916, 1917, 1919 y 1922): "Inventarios aragoneses de los siglos XIV y XV", Boletín de la Real Academia Española, 2, pp. 85-97, 219-224, 341-352, 548-559, 707-711, 3, pp. 89-92, 224-225, 359-365, 4, pp. 207-223, 342-355, 517531, 6, pp. 735-744, 9, pp. 118-134, 262-270.

Sesma, José Ángel y Ángeles Líbano (1982): Léxico del comercio medieval en Aragón (siglo XV), Zaragoza, Institución Fernando el Católico.

Terlingen, Juan (1943): Los italianismos en español. Desde la formación del idioma hasta principios del siglo XVII, Amsterdam.

Terrado Pablo, Javier (1991): La lengua de Teruel a fines de la Edad Media, Teruel, Instituto de Estudios Turolenses. Diputación Provincial de Teruel.

TerReros y Pando, Esteban de (1987): Diccionario castellano con las voces de ciencias y artes, Madrid, Arco/Libros.

TIlander, GunNar (1935): "Fueros aragoneses desconocidos, promulgados a consecuencia de la gran peste de 1348", Revista de Filología Española, 22, pp. 1-33 y 113-152.

Viudas CAMARASA, Antonio (1978): "Léxico dialectal de la Llitéra (Ganadería, fenómenos atmosféricos y reino animal)", Anuario de estudios filológicos, 7, pp. 295-324.

Zamora Vicente, Alonso (1989): Dialectología española, Madrid, Gredos, $2^{\mathrm{a}}$ ed. 